\title{
Medo de cair e qualidade de vida em idosos com catarata
}

\author{
Fear of falling and quality of life in elderly with cataract
}

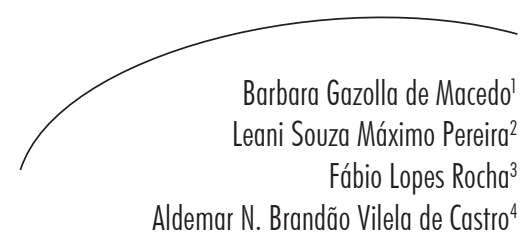

Resumo

Introducão: $\mathrm{O}$ medo de cair é definido como baixa autoeficácia ou confiança no próprio equilíbrio para evitar quedas, causando declínio no desempenho físico e funcional, alterações no equilíbrio e na marcha dos idosos, tendo impacto negativo na qualidade de vida. Vários estudos têm identificado o déficit visual como fator contributivo para a ocorrência de quedas e do medo de cair em idosos. Objetivos: Avaliar a associação entre o medo de cair e as alterações na visão funcional e na qualidade de vida relacionada à visão de idosos com catarata. Métodos: Trata-se de estudo observacional com 139 idosos com catarata $(71,4 \pm 6,2$ anos). A qualidade de vida relacionada à visão foi avaliada pelo Visual Function Questionnaire (VFQ) e a preocupação com a possibilidade de quedas foi avaliada pela Falls Efficacy Scale International (FES-I-Brasil). Resultados: Idosos que relataram algum grau de medo de cair apresentaram médias mais altas na escala FES-IBrasil, quando comparados aos que não o relataram, indicando maior preocupação com a possibilidade de cair ( $\mathrm{p}=0,000$; Kruskal-Wallis). Com relação à visão funcional (VFQ) e à qualidade de vida relacionada à visão, quanto maior o medo de cair, piores eram a visão e a qualidade de vida ( $\mathrm{p}=0,003$; Kruskal-Wallis). Conclusão: Idosos com catarata e com relato de medo de cair apresentaram menor confiança no seu equilíbrio para evitar quedas e pior qualidade na visão funcional e na qualidade de vida relacionada à visão.

\section{Abstract}

Introduction: Fear of falling is defined as low self-efficacy or self-confidence in one's own balance to avoid falls. It causes limitations in physical and functional performance, balance and walking impairment, and has negative impact on quality of life. Many research works have identified visual impairment as one of the factors that contribute

\footnotetext{
Clínica de Fisioterapia. Instituto da Previdência dos Servidores do Estado de Minas Gerais. Belo Horizonte, MG, Brasil.

2 Departamento de Fisioterapia, Escola de Educação Física, Fisioterapia e Terapia Ocupacional. Universidade Federal de Minas Gerais. Belo Horizonte, MG, Brasil.

3 Clínica Psiquiátrica. Instituto da Previdência dos Servidores do Estado de Minas Gerais. Belo Horizonte, MG, Brasil.

4 Clínica Oftalmológica. Instituto da Previdência dos Servidores do Estado de Minas Gerais. Belo Horizonte, MG, Brasil.
}

Palavras-chave: Medo de Cair. Visão, Ocular. Qualidade de Vida. Idoso. 
to falls and fear of falling. Objectives: Evaluate the association between fear of falling and visual function impairment and the related-vision quality of life in elderly patients with cataract. Methods: Observational study conducted with 139 elderly patients with cataract (71.4⒍ 6.2 years). Vision-related quality of life was assessed through the Visual Function Questionnaire (VFQ). Interviews were made by means of the Falls Efficacy Scale International (FES-I Brazil) to evaluate the concern with falling. Results: Elderly patient with fear of falling had higher averages in FES-I-Brazil than other elderly patients ( $\mathrm{p}=0.000$; Kruskal-Wallis). The greater the fear of falling, the worst were the visual function (VFQ) and the related-vision quality of life ( $\mathrm{p}=0,003$; Kruskal-Wallis). Conclusion: Elderly patients with cataract and fear of falling had less self-confidence in their balance to avoid falls and worst functional vision and related-vision quality of life.
Key words: Fear of Falling. Vision, Ocular. Quality of Life. Elderly.

\section{INTRODUÇÃO}

O medo de cair é definido como baixa autoeficácia ou confiança no próprio equilíbrio para evitar quedas, causando declínio no desempenho físico e funcional, alterações no equilíbrio e na marcha dos idosos e impacto negativo na qualidade de vida. ${ }^{1-5}$ Quedas, por sua vez, representam um grande problema social, por se relacionarem com o declínio físico do idoso e por trazerem consequências psicossociais como depressão, ansiedade e medo de cair. A prevalência do medo de cair é maior em mulheres, idosos longevos, naqueles com história prévia de quedas, mobilidade reduzida e fragilidade. ${ }^{2-5}$

A avaliação do medo de cair é realizada por questionamentos simples com respostas dicotômicas e subjetivas (ex: ter ou não medo de cair). Atualmente, a literatura sugere o uso de escalas específicas que avaliam a influência do medo de cair no desempenho funcional dos idosos. Uma das escalas mais utilizadas é a Falls Efficacy Scale (escala de eficácia em quedas), instrumento já traduzido e adaptado para o Brasil. ${ }^{2,45}$

Vários estudos têm identificado o déficit visual como fator contributivo para a ocorrência de quedas e do medo de cair em idosos. Idosos com baixa acuidade visual tendem a diminuir suas atividades básicas e instrumentais de vida diária. ${ }^{6-10} \mathrm{~A}$ presença de alterações visuais, seja na acuidade, no contraste, no ofuscamento ou na percepção de profundidade, como é o caso da catarata, é relatada como fator que aumenta a morbidade e a mortalidade em idosos. ${ }^{11}$

A catarata é uma das doenças que mais acometem os idosos, sendo definida como qualquer opacidade do cristalino que difrate a luz, acarretando efeito negativo na visão. De acordo com a Organização Mundial da Saúde, a catarata é a desvantagem visual mais frequente no mundo. É considerada a principal causa de cegueira, sendo responsável por cerca de $50 \%$ dos 50 milhões de casos. ${ }^{11}$

O objetivo deste estudo foi avaliar e verificar a associação entre o medo de cair e as alterações na visão funcional e na qualidade de vida relacionada à visão de idosos com catarata.

\section{METODOLOGIA}

Trata-se de estudo observacional do qual participaram 139 idosos referenciados por médicos de uma clínica oftalmológica, entre os meses de outubro de 2006 e julho de 2007. Realizou-se cálculo amostral com 50 participantes. Considerando um poder mínimo de $80 \%$ para detectar as diferenças das médias entre os grupos e um nível de significância de 5\%, seria necessária a inclusão de 121 participantes. Para tal, foi utilizado o teste do qui-quadrado considerando três graus de liberdade. Os seguintes critérios de inclusão foram obedecidos: ter 60 anos ou mais, diagnóstico de catarata, e deambular de forma independente e sem auxílio de órteses. Foram excluídos: pacientes sem 
deambulação independente, com claudicação, presença de doenças neurológicas, labirintites, hipotensão ortostática sintomática, alterações cognitivas, em pós-operatórios recentes, com qualquer artroplastia ou osteossínteses dos membros inferiores, doenças reumatológicas e ortopédicas ativas, queixas álgicas, fraqueza dos membros inferiores, pacientes com cirurgias oftalmológicas prévias e glaucoma.

Para a seleção da amostra, quanto aos aspectos cognitivos, foi aplicado o mini-exame do estado mental. ${ }^{12}$ Em seguida, realizaram-se testes de força muscular manual para os grupos musculares dos membros inferiores, a fim de excluir idosos com fraqueza muscular. ${ }^{13}$

O estudo foi aprovado pelo Comitê de Ética e Pesquisa do Instituto da Previdência dos Servidores do Estado de Minas Gerais, sob o $n^{\circ}$ 218/06, e todos os participantes assinaram o Termo de Consentimento Livre e Esclarecido.

\section{Instrumentos e procedimentos para a coleta dos dados}

Os idosos responderam inicialmente a um questionário clínico sociodemográfico desenvolvido pelos pesquisadores (sexo, idade, escolaridade, doenças associadas, uso de medicamentos, história de quedas e presença de medo de cair).

A qualidade de vida e a visão funcional foram avaliadas por meio do questionário de visão funcional (VFQ), ${ }^{14}$ que apresenta 25 questões agrupadas em 12 subdomínios: saúde geral, visão, dor ocular, atividades para perto, atividades para longe, aspectos sociais, saúde mental, atividades de vida diária, dependência, capacidade para dirigir automóveis, visão de cores e visão periférica. A pontuação máxima obtida no questionário é de 100 pontos. Quanto maior a pontuação, melhores a visão e a qualidade de vida relacionada à visão.

Para avaliação do medo de cair, os idosos foram questionados sobre essa possibilidade e as respostas foram estratificadas nas seguintes categorias: não tenho medo, pouco, moderado e muito medo de cair. Também se utilizou a Escala Internacional de Eficácia em Quedas (FES-I-Brasil), ${ }^{4}$ que apresenta questões sobre a preocupação com a possibilidade de cair ao realizar 16 atividades diferentes: (1) limpando a casa; (2) vestindo ou tirando a roupa; (3) preparando refeições simples; (4) tomando banho; (5) indo às compras; (6) sentando ou levantando de uma cadeira; (7) subindo ou descendo escadas; (8) caminhando pela vizinhança; (9) pegando algo acima de sua cabeça ou do chão; (10) ir atender ao telefone antes que pare de tocar; (11) andando sobre superfície escorregadia; (12) visitando um amigo ou parente; (13) andando em lugares cheios de gente; (14) caminhando sobre superfície irregular; (15) subindo ou descendo uma ladeira; e (16) indo a uma atividade social. A pontuação máxima obtida é de 64 pontos. Quanto maior a pontuação, maior a preocupação com a possibilidade de cair. Os testes foram aplicados na clínica oftalmológica, por dois fisioterapeutas previamente treinados em um estudo piloto.

\section{Análise estatística}

Para a caracterização da amostra, utilizou-se a análise descritiva; e para avaliar a associação entre o medo de cair e demais variáveis, foram utilizados o teste não paramétrico de KruskalWallis, o teste de Mann-Whitney e qui-quadrado. O programa estatístico utilizado foi o SPSS - Statistical Package for the Social Sciences, versão 12, instalado em ambiente Windows, sendo estabelecido um $\alpha=0,05$.

\section{RESULTADOS}

Foram avaliados 139 idosos, com média de idade de 71,4 anos $( \pm 6,2)$, com diagnóstico de catarata. Destes, 64\% eram do sexo feminino, $69,1 \%$ apresentavam doenças cardiovasculares e $50,4 \%$ relataram algum grau de medo de cair. A maioria dos idosos $(58,2 \%)$ recebeu o diagnóstico de catarata há pelo menos um ano. Os resultados descritivos das variáveis qualitativas e quantitativas estão descritos na tabela 1. 
Tabela 1 - Análise descritiva das variáveis qualitativas da amostra pesquisada. Belo Horizonte-MG, 2007.

\begin{tabular}{|c|c|c|c|}
\hline Variáveis descritivas & Categorias & $\mathrm{n}$ & $\%$ \\
\hline \multirow[t]{2}{*}{ Sexo } & Feminino & 89 & 64,0 \\
\hline & Masculino & 50 & 36,0 \\
\hline \multirow[t]{8}{*}{ Escolaridade } & Analfabeto & 8 & 5,8 \\
\hline & $1^{\circ}$ grau incompleto & 65 & 46,8 \\
\hline & $1^{\circ}$ grau completo & 11 & 7,9 \\
\hline & $2^{\circ}$ grau incompleto & 1 & 0,7 \\
\hline & $2^{\circ}$ grau completo & 35 & 25,2 \\
\hline & Superior incompleto & 2 & 1,4 \\
\hline & Superior completo & 17 & 12,2 \\
\hline & Cardiovascular & 96 & 69,1 \\
\hline \multicolumn{4}{|l|}{ Problemas de saúde } \\
\hline & Osteomuscular & 26 & 18,7 \\
\hline & Gastrointestinal & 21 & 15,1 \\
\hline & Geniturinário & 5 & 3,6 \\
\hline & Neurológico & 2 & 1,4 \\
\hline & Pulmonar & 3 & 2,2 \\
\hline & Psiquiátrico & 12 & 8,6 \\
\hline & Dermatológico & 2 & 1,4 \\
\hline & Auditivo & 5 & 3,6 \\
\hline & Outros & 18 & 12,9 \\
\hline \multirow[t]{2}{*}{ Quedas no último ano } & $\operatorname{Sim}$ & 58 & 41,7 \\
\hline & Não & 81 & 58,3 \\
\hline \multirow[t]{4}{*}{ Tem medo de cair } & Não & 69 & 49,6 \\
\hline & Um pouco & 20 & 14,4 \\
\hline & Moderado & 24 & 17,3 \\
\hline & Muito & 26 & 18,7 \\
\hline
\end{tabular}


A tabela 2 mostra a distribuição, de acordo com o sexo, da presença de quedas no último ano e do relato do medo de cair. O grupo das mulheres idosas apresentou maior número de quedas no último ano e maior relato de medo, quando comparadas ao grupo masculino $(\mathrm{p}=0,000)$. As médias do desempenho nos instrumentos de avaliação foram FES-I-Brasil $=24,1 \pm 6,8$ e VFQ= 77,7 $\pm 15,9$.
O grupo de mulheres idosas apresentou maior preocupação com o medo de cair (FESI-Brasil, média $=26,2 \pm 7,1)$, e piores visão e qualidade de vida relacionada à visão (VFQ, média $=76,3 \pm 15,1)$, quando comparado ao grupo masculino (FES-I-Brasil, média $=20,3 \pm 3,9$ ), $\mathrm{p}=0,000$ e VFQ, média $=80,1 \pm 17,1$, teste de Mann-Whitney, $\mathrm{p}=0,000$.

Tabela 2 - A associação entre o sexo e demais variáveis qualitativas da amostra pesquisada. Belo Horizonte-MG, 2007.

\begin{tabular}{|c|c|c|c|c|c|}
\hline \multirow[b]{2}{*}{ Variáveis } & \multirow[b]{2}{*}{ Categorias } & \multicolumn{2}{|c|}{ Sexo } & \multirow[b]{2}{*}{ Total } & \multirow[b]{2}{*}{ Valor $\mathrm{p}$} \\
\hline & & $\begin{array}{l}\text { Masculino } \\
\quad(\mathrm{n}=50)\end{array}$ & $\begin{array}{c}\text { Feminino } \\
(\mathrm{n}=89)\end{array}$ & & \\
\hline \multirow[t]{4}{*}{ Queda no último ano } & Não & 40 & 41 & 81 & 0,000 \\
\hline & & $80,0 \%$ & $46,1 \%$ & $58,3 \%$ & \\
\hline & $\operatorname{Sim}$ & 10 & 48 & 58 & \\
\hline & & $20,0 \%$ & $53,9 \%$ & $41,7 \%$ & \\
\hline \multirow[t]{8}{*}{ Tem medo de cair } & Não & 38 & 31 & 69 & 0,000 \\
\hline & & $76,0 \%$ & $34,8 \%$ & $49,6 \%$ & \\
\hline & Um pouco & 2 & 18 & 20 & \\
\hline & & $4,0 \%$ & $20,2 \%$ & $14,4 \%$ & \\
\hline & Mais ou menos & 6 & 18 & 24 & \\
\hline & & $12,0 \%$ & $20,2 \%$ & $17,3 \%$ & \\
\hline & Muito & 4 & 22 & 26 & \\
\hline & & $8,0 \%$ & $24,7 \%$ & $18,7 \%$ & \\
\hline
\end{tabular}

Teste qui-quadrado.

Idosos que relataram algum grau de medo de cair apresentaram médias mais altas na escala FES-I-Brasil, quando comparados aos que não relataram, indicando maior preocupação com a possibilidade de cair $(\mathrm{p}=0,000)$. Com relação à visão funcional (VFQ) e à qualidade de vida relacionada à visão, quanto maior o medo de cair, piores eram a visão e a qualidade de vida $(p=0,003)$, segundo a tabela 3. 
Tabela 3 - Análise das variáveis: escala de eficácia de quedas (FES-I-Brasil) e questionário de visão funcional (VFQ), de acordo com o medo de cair. Belo Horizonte-MG, 2007.

\begin{tabular}{|c|c|c|c|c|c|c|c|c|}
\hline Variáveis & $\begin{array}{l}\text { Tem medo } \\
\text { de cair }\end{array}$ & $\mathrm{n}$ & Média & $\begin{array}{l}\text { Desvio } \\
\text { Padrão }\end{array}$ & Mín. & Mediana & Max. & $\begin{array}{l}\text { Valor } \\
\mathrm{p}^{*}\end{array}$ \\
\hline \multicolumn{9}{|l|}{ FES-I-Brasil } \\
\hline \multirow[t]{4}{*}{ Escore total } & Não & 69 & 21,3 & 5,6 & 16,0 & 20,0 & 44,0 & 0,000 \\
\hline & Um pouco & 20 & 24,0 & 4,7 & 19,0 & 21,5 & 35,0 & \\
\hline & $\begin{array}{l}\text { Mais ou } \\
\text { menos }\end{array}$ & 24 & 27,0 & 8,0 & 17,0 & 25,5 & 48,0 & \\
\hline & Muito & 26 & 29,0 & 6,1 & 20,0 & 28,0 & 40,0 & \\
\hline \multicolumn{9}{|l|}{ VFQ } \\
\hline \multirow[t]{4}{*}{ Escore total } & Não & 69 & 81,1 & 14,6 & 37,5 & 86,0 & 99,0 & 0,003 \\
\hline & Um pouco & 20 & 79,6 & 17,2 & 28,3 & 84,5 & 94,6 & \\
\hline & $\begin{array}{l}\text { Mais ou } \\
\text { menos }\end{array}$ & 24 & 72,4 & 15,3 & 41,3 & 73,9 & 94,6 & \\
\hline & Muito & 26 & 71,9 & 16,6 & 23,9 & 76,1 & 95,4 & \\
\hline
\end{tabular}

*Teste não paramétrico de Kruskal-Wallis.

\section{DISCUSSÃO}

Neste estudo a amostra foi composta, em sua maioria, pelo sexo feminino (64,6\%), mostrando o processo de feminização da velhice em nosso país. Esse fato pode ser explicado pelos fatores culturais e/ou sociais, que fazem com que a mulher procure mais os serviços de saúde. ${ }^{15,16}$

Costa e colaboradores ${ }^{17}$ conduziram estudo transversal com 419 idosos da comunidade Belga, com o objetivo de avaliar a frequência do medo de cair e a restrição de atividades. A maioria dos idosos com relato de medo de cair era do sexo feminino $(68,3 \%)$. Outro resultado importante do estudo foi referente ao histórico de quedas: $82 \%$ dos idosos com duas ou mais quedas no último ano relataram medo de cair; $70 \%$ com uma queda relataram medo e $50 \%$ dos idosos que não caíram no último ano relataram medo de quedas. Neste estudo, as mulheres, além de relatarem mais medo de cair, também apresentaram maior número de quedas no último ano.

Metade $(50,4 \%)$ dos pacientes com catarata deste estudo relatou algum grau de medo de cair, classificado como: pouco, moderado e muito. Esse resultado está de acordo com a literatura, a qual relata importante associação entre os problemas visuais e a presença do medo de cair. ${ }^{18-23}$ Wang e colaboradores ${ }^{24}$ também avaliaram o medo de cair em idosos com déficit visual: $50 \%$ do grupo de idosos com déficit visual apresentaram limitação de suas atividades diárias devido ao medo de cair e apenas 16\% do grupo controle com visão normal limitaram suas atividades diárias devido ao medo de quedas. É importante ressaltar que esse estudo não avaliou idosos com catarata, apenas idosos com glaucoma e degeneração macular. Outro estudo relevante de uma coorte da Finlândia com 434 idosas da comunidade também encontrou 
associação entre déficit sensoriais, como a visão, e a presença do medo de cair. ${ }^{25}$

Alguns autores sugerem que o medo de cair deve ser avaliado por escalas específicas, pois assim será possível avaliar melhor o desempenho dos idosos, quando comparadas com questionários subjetivos e dicotômicos..$^{2-5}$ Dessa forma, optou-se neste estudo por avaliar o medo de cair com respostas estratificadas em categorias e um instrumento mais específico, como a FES-I Brasil.

As atividades da FES-I-Brasil que causaram maior preocupação em relação à possibilidade de quedas entre os idosos deste estudo foram aquelas que exigiam elevado nível funcional (itens 04, 07, $11,14,15)$. Esse resultado se deve à amostra deste estudo ser composta por idosos da comunidade, independentes para as atividades de vida diária. Observou-se, também, que idosos que relataram medo de quedas tiveram pior desempenho na escala FES-I-Brasil, quando comparados aos que não relataram medo. Esse resultado foi estatisticamente significativo $(p=0,00)$ e mostra que a FES-I-Brasil pode ser um instrumento adequado para avaliar o medo de cair em idosos.

Encontrou-se associação estatisticamente significativa entre medo de cair e visão funcional $(\mathrm{p}=0,03)$. Quanto maior o relato de medo de cair pelos idosos, pior era a visão funcional. A avaliação da visão funcional não engloba somente a acuidade visual; ela avalia a sensibilidade à luz, aos contrastes, a percepção de cores, a visão periférica, além de processos interpretativos. ${ }^{26-31}$ O questionário VFQ foi utilizado, nesta amostra, para identificar de forma subjetiva as alterações na função visual e na qualidade de vida.

Apesar de a literatura citar a importância da avaliação da visão como um todo, vários estudos avaliam apenas a associação da acuidade visual e a presença de quedas. ${ }^{6-10,26,27,29,32}$ Poucos são os estudos que avaliam a baixa visão funcional e as consequências do evento "quedas", como o medo de cair. ${ }^{18,19,21}$ É importante avaliar a associação do déficit na visão funcional e o medo de cair, para que não se subestime o verdadeiro poder dessa associação.

Estudo multicêntrico realizado com 439 idosos com catarata também utilizou como instrumento de medida para visão e qualidade de vida o questionário VFQ. Os autores relataram a associação entre o déficit visual e o comprometimento na qualidade de vida em idosos com catarata. Maior número de quedas no grupo com baixa visão e pior qualidade de vida também foram encontrados, mas os pesquisadores não avaliaram a presença do medo de cair. ${ }^{33}$

Com relação à qualidade de vida relacionada à visão, observou-se neste estudo que quanto maior o relato de medo de quedas, pior a qualidade de vida desses idosos. Esses dados estão de acordo com a literatura, que relata o impacto negativo na qualidade de vida dos idosos com problemas visuais e medo de cair. . $^{-5,14,15,18,21}$

No entanto, o presente estudo apresenta algumas limitações. Por ser um trabalho observacional, não tem efeito de causalidade. Outras variáveis que pudessem interferir no medo de cair não foram avaliadas como, por exemplo, outros déficits sensoriais.

\section{CONCLUSÃO}

Idosos com catarata e com relato de medo de cair podem apresentar menor confiança no seu equilíbrio para evitar quedas e pior qualidade na visão funcional e na qualidade de vida relacionada à visão. Muitos problemas relacionados à visão em idosos são passíveis de correção e tratamento, e devem ser incentivados, seja por meio das órteses ou mesmo da extração da catarata.

Sugerem-se, assim, novos estudos comparativos para investigar o medo de cair e a qualidade de vida em idosos antes e após a cirurgia de catarata. 


\section{REFERÊNCIAS}

1. Bloem BR, Steijns JA, Smits-Engelsman BC. An update on falls. Curr Opin Neurol 2003;16(1):15-26.

2. Legters K. Fear of falling. Phys Ther 2002;82(3):264-72.

3. Powell LE, Myers AM. The Activities-specific Balance Confidence (ABC) Scale. J Gerontol A Biol Sci Med Sci 1995;50A(1):M28-34.

4. Camargos FFO. Adaptação transcultural e avaliação das propriedades psicométricas da falls efficacy ScaleInternational: um instrumento para avaliar medo de cair em idosos [tese]. Belo Horizonte: UFMG; 2007.

5. Tinetti ME, Richman D, Powell L. Falls efficacy as a measure of fear of falling. J Gerontol 1990;45(6):P239-43.

6. Lee HK, Scudds RJ. Comparison of balance in older people with and without visual impairment. Age Ageing 2003;32(6):643-9.

7. Daubney ME, Culham EG. Lower-extremity muscle force and balance performance in adults aged 65 years and older. Phys Ther 1999;79(12):1177-85.

8. Jack CI, Smith T, Neoh C, Lye M, McGalliard JN. Prevalence of low vision in elderly patients admitted to an acute geriatric unit in Liverpool: elderly people who fall are more likely to have low vision. Gerontology 1995;41(5):280-5.

9. Keller BK, Morton JL, Thomas VS, Potter JF. The effect of visual and hearing impairments on functional status. J Am Geriatr Soc 1999;47(11):1319-25.

10. Maeda A, Nakamura K, Otomo A, Higuchi S, Motohashi Y. Body support effect on standing balance in the visually impaired elderly. Arch Phys Med Rehabil 1998;79(8):994-7.

11. Zacharias LC, Graziano RM, Oliveira BFT, Hatanaka M, Cresta FB, Kara-José N. A campanha da catarata atrai pacientes da clínica privada? Arq Bras Oftalmol 2002;65(5):557-61.

12. Bertolucci PHF, Brucki SMD, Compacci SR, Juliano Y. O mini-exame do estado mental em uma população geral: impacto da escolaridade. Arq Neuropsiquiatr 1994;52(1):1-7.

13. Kendall FP, McCreary EK. Músculos, provas e funções. 3. ed. São Paulo: Manole; 1987.

14. Ferraz EVAP, Lima CA, Cella W, Arieta CEL. Adaptation of the questionnaire of the assessment of life quality for application with patients with cataracts. Arq Bras Oftalmol 2002;65:293-8.
15. Oliveira RSCS, Temporini ER, Kara-José N, Carricondo PC, Kara-José AC. Perceptions of patients about cataract. Clinics 2005;60(6):455-60.

16. Laurenti R, Jorge MHPM, Gotlieb SLD. Perfil epidemiológico da morbi-mortalidade masculina. Ciênc Saúde Coletiva 2005;10(1):35-46.

17. Costa EM, Pepersack T, Levêque A. Fear of falling and associated activity restriction in older people. Results of a cross-sectional study conducted in a Belgian town. Arch Public Health 2012;70(1):1

18. Salive ME, Guralnik J, Glynn RJ, Christen W, Wallace RB, Ostfeld AM. Association of visual impairment with mobility and physical function. J Am Geriatr Soc 1994;42(3):287-92.

19. Lamoureux E, Gadgil S, Pesudovs K, Keeffe J, Fenwick E, Dirani M, et al. The relationship between visual function, duration and main causes of vision loss and falls in older people with low vision. Graefes Arch Clin Exp Ophthalmol 2010;248(4):527-33.

20. Lord SR, Smith ST, Menant JC. Vision and falls in older people: risk factors and intervention strategies. Clin Geriatr Med 2010;26(4):569-81.

21. Dillon CF, Gu Q, Hoffman HJ, Ko CW. Vision, hearing, balance, and sensory impairment in Americans aged 70 years and over: United States, 1999-2006. NCHS Data Brief 2010;(31):1-8.

22. Black A, Wood J. Vision and falls. Clin Exp Optom 2005;88(4):212-22.

23. West CG, Gildengorin G, Haegerstrom-Portnoy G, Schneck ME, Lott L, Brabyn JA. Is vision function related to physical functional ability in older adults? J Am Geriatr Soc 2002;50(1):136-45.

24. Wang MY, Rousseau J, Boisjoly H, Schmaltz H, Kergoat MJ, Moghadaszadeh S, et al. Activity limitation due to a fear of falling in older adults with eye disease. Invest Ophthalmol Vis Sci 2012;53(13):7967-72.

25. Viljanen A, Kulmala J, Rantakokko M, Koskenvuo M, Kaprio J, Rantanen T. Fear of falling and coexisting sensory difficulties as predictors of mobility decline in older women. J Gerontol A Biol Sci Med Sci 2012;67(11):1230-7.

26. Schwartz S, Segal O, Barkana Y, Schwesig R, Avni I, Morad Y. The effect of cataract surgery on postural control. Invest Ophthalmol Vis Sci 2005;46(3):920-4

27. Anand V, Buckley J, Scally A, Elliott DB. The effect of refractive blur on postural stability. Ophthalmic Physiol Opt 2002;22(6):528-34. 
28. Anand V, Buckley JG, Scally A, Elliott DB. Postural stability in the elderly during sensory perturbations and dual tasking: the influence of refractive blur. Invest Ophthalmol Vis Sci 2003;44(7):2885-91.

29. Lord SR, Menz HB. Visual contributions to postural stability in older adults. Gerontology 2000;46(6):306-10.

30. Teasdale N, Simoneau M. Attentional demands for postural control: the effects of aging and sensory reintegration. Gait Posture 2001;14(3):203-10.

31. Huang M, Burgess R, Weber M, Greenwald N. Performance of balance impaired elders on three balance tests under two visual conditions. J Geriatr Phys Ther 2006;29(1):5-9.

32. Owsley C, McGwin G Jr. Association between visual attention and mobility in older adults. J Am Geriatr Soc 2004;52(11):1901-6.

33. Yamada M, Mizuno Y, Miyake Y; Cataract Survey Group of the National Hospital Organization of Japan. A multicenter study on the health-related quality of life of cataract patients: baseline data. Jpn J Ophthalmol 2009;53(5):470-6. 